\title{
Interval projections of self-similar sets
}

\author{
Ábel Farkas
}

\begin{abstract}
We show that if $K$ is a self-similar 1-set that is not contained in a line and either satisfies the strong separation condition or is defined via homotheties then there are at most finitely many lines through the origin such that the projection of $K$ onto them is an interval.
\end{abstract}

\section{Introduction}

\subsection{Overview}

The measure theory of projections of 'fractals' has gained much attention in the past few decades. A seminal result is that for a Borel set $K \subseteq \mathbb{R}^{2}$ if $\operatorname{dim}_{H}(K)>1$ then

$$
\mathcal{H}^{1}\left(\Pi_{M}(K)\right)>0
$$

for almost all lines $M$, where $\operatorname{dim}_{H}$ denotes the Hausdorff dimension, $\mathcal{H}^{s}$ denotes the $s$-dimensional Hausdorff measure and $\Pi_{M}: \mathbb{R}^{2} \longrightarrow M$ denotes the orthogonal projection onto $M$. This was proved by Marstrand [8], and was generalized to higher dimensions by Mattila [10. When $\operatorname{dim}_{H}(K)<1$ then $\mathcal{H}^{1}\left(\Pi_{M}(K)\right)=0$ for every line $M$ since projection does not increase the Hausdorff dimension. In the critical case when $\operatorname{dim}_{H}(K)=1$ two things can happen. A set $K \subseteq \mathbb{R}^{d}$ is called an s-set if $0<\mathcal{H}^{s}(K)<\infty$. We call a 1-set $K$ purely 1-unrectifiable if $\mathcal{H}^{1}(K \cap M)=0$ for every differentiable 1-manifold $M$. It was shown by Besicovitch [1] and generalised to higher dimensions by Federer [5] that for a 1-set $K \subseteq \mathbb{R}^{2}$

$$
\mathcal{H}^{1}\left(\Pi_{M}(K)\right)=0
$$

for almost all lines $M$ if and only if $K$ is purely 1-unrectifiable. If $K$ is not purely 1-unrectifiable then $\mathcal{H}^{1}\left(\Pi_{M}(K)\right)>0$ for all but at most one lines $M$.

When $K \subseteq \mathbb{R}$ is a dynamically defined set often the case is that $\mathcal{H}^{1}(K)>0$ if and only if $K$ contains an interval. For example, if $K \subseteq \mathbb{R}$ is a self-similar set satisfying the 'open set condition' then $\mathcal{H}^{1}(K)>0$ if and only if $K$ contains an interval [12, Corollary 2.3]. Whether the statement still holds without assuming the open set condition is an intriguing, still open question. If $K \subseteq \mathbb{R}^{2}$ is a self-similar 1-set satisfying the open set condition then for a line $M$ we have that $\mathcal{H}^{1}\left(\Pi_{M}(K)>0\right.$ if and only if $\Pi_{M}(K)$ contains an interval (see [4, Thm 1.1; Thm 1.5], [13] and [12, Corollary 2.3]).

In this paper we are concerned about the situation when the projection $\Pi_{M}(K)$ not only contains an interval but is an interval itself. The unit semicircle in the plane is a 1-set and the projection of it onto every line is an interval. Falconer and Fraser [3] studied the visible part of self-similar sets that project onto an interval in every direction. Our main results show that under some natural assumptions on a self-similar 1-set $K$ there are at most finitely many lines $M$ such that $\Pi_{M}(K)$ is an interval in contrast with the example of the unit semicircle which is a self-conformal set. This is the case when $K$ satisfies the 'strong separation condition'. We also show that we can drop the assumption of the strong separation condition if we assume that every defining map is a homothety. In the last section we give examples of self-similar sets with several interval projections. Finally, we establish an invariance property of the moment of inertia of a self-similar 1-set with several interval projections.

\subsection{Definitions and notations}

For integers $0 \leq l<d$ and for an $l$-dimensional affine subspace $M \subseteq \mathbb{R}^{d}$ we denote the orthogonal projection onto $M$ by $\Pi_{M}: \mathbb{R}^{d} \longrightarrow M$. Throughout the paper we consider $\Pi_{M}$ as a mapping into $\mathbb{R}^{l}=M$. 
Definition 1.1. Let $M \subseteq \mathbb{R}^{2}$ be a line through the origin and $K \subseteq \mathbb{R}^{2}$ be an arbitrary set. We call the projection $\Pi_{M}: \mathbb{R}^{2} \longrightarrow M$ an interval projection of $K$ if $\Pi_{M}(K)$ is an interval with the $M=\mathbb{R}$ identification. For a single point $x \in \mathbb{R}$ we consider $\{x\}$ to be the closed interval $[x, x]$ of length 0 .

A self-similar iterated function system (SS-IFS) in $\mathbb{R}^{d}$ is a finite collection of maps $\left\{S_{i}\right\}_{i=1}^{m}$ from $\mathbb{R}^{d}$ to $\mathbb{R}^{d}$ such that all the $S_{i}$ are contracting similarities. The attractor of the SS-IFS is the unique nonempty compact set $K$ such that $K=\bigcup_{i=1}^{m} S_{i}(K)$. The attractor of an SS-IFS is called a self-similar set. We say that the SS-IFS $\left\{S_{i}\right\}_{i=1}^{m}$ satisfies the strong separation condition (SSC) if the $\left\{S_{i}(K)\right\}_{i=1}^{m}$ are a disjoint. Every $S_{i}$ can be uniquely decomposed as

$$
S_{i}(x)=r_{i} T_{i}(x)+v_{i}
$$

for all $x \in \mathbb{R}^{d}$, where $0<r_{i}<1, T_{i}$ is an orthogonal transformation and $v_{i} \in \mathbb{R}^{d}$ is a translation vector. Let $\mathcal{T}$ denote the group generated by the orthogonal transformations $\left\{T_{i}\right\}_{i=1}^{m}$. We denote the set $\{1,2, \ldots, m\}$ by $\mathcal{I}$. Let $\mathbf{i}=\left(i_{1}, \ldots, i_{k}\right) \in \mathcal{I}^{k}$ i.e. a $k$-tuple of indices. Then we write $S_{\mathbf{i}}=S_{i_{1}} \circ \ldots \circ S_{i_{k}}$ and $K_{\mathbf{i}}=S_{\mathbf{i}}(K)$. Since the similarities are decomposed as in (2) we write $r_{\mathbf{i}}=r_{i_{1}} \cdot \ldots \cdot r_{i_{k}}$ and $T_{\mathbf{i}}=T_{i_{1}} \circ \ldots \circ T_{i_{k}}$.

\subsection{Finiteness of interval projections}

In this section we list the main results of this paper. The proofs are provided in Section 3 and Section 4 .

We provide examples, Example 5.5, of self-similar sets with Hausdorff dimension arbitrarily close to 1 such that projection of them onto every line is an interval. However, when the Hausdorff dimension is 1 we prove that there are only finitely many such lines. We also provide an example of a totally disconnected, compact, non-self-similar set of Hausdorff dimension 1 that projects onto an interval in every direction (see Example [5.6).

Theorem 1.2. Let $\left\{S_{i}\right\}_{i=1}^{m}$ be a self-similar iterated function system in $\mathbb{R}^{2}$ with attractor $K$ such that $\left\{S_{i}\right\}_{i=1}^{m}$ satisfies the strong separation condition and $K$ is a 1-set. Then there are at most finitely many lines through the origin such that the orthogonal projection onto them are interval projections of $K$.

The 1-dimensional Sierpinski triangle $K_{\triangle}$ (see Example 5.1) is a self-similar 1-set and the usual SS-IFS for $K_{\triangle}$ satisfies the SSC and $|\mathcal{T}|=1$, where |.| denotes the cardinality of a set. Hence the projection onto every line is a self-similar set with 'similarity dimension' 1 and so by [12, Corollary (2.3)] the projection has positive $\mathcal{H}^{1}$-measure if and only if the projection contains an interval. Kenyon [7] showed that this occurs exactly for a countable and dense set of lines through the origin. So there exists a dense set of lines through the origin onto which the projection contains an interval, but the projection onto at most finitely many of them is an interval projection by Theorem 1.2. One can show that $K_{\triangle}$ has exactly three interval projections. In Example 5.2 and Example 5.4 we provide examples of self-similar 1-sets such that the projection of them onto four different lines are intervals.

While Theorem 1.2 requires the SSC, we would like to eliminate this separation condition but to do so we need a further assumption on $\mathcal{T}$. A similarity $S: \mathbb{R}^{d} \longrightarrow \mathbb{R}^{d}$ is called a homothety if there are $r \in \mathbb{R} \backslash\{0\}$ and $v \in \mathbb{R}^{2}$ such that $S(x)=r x+v$ for every $x \in \mathbb{R}^{d}$.

Theorem 1.3. Let $\left\{S_{i}\right\}_{i=1}^{m}$ be a self-similar iterated function system in $\mathbb{R}^{2}$ with attractor $K$ such that all $S_{i}$ are homotheties and $K$ is a 1 -set that is not contained in any line. Then there are at most finitely many lines through the origin such that the orthogonal projection onto them are interval projections of $K$.

Remark 1.4. The reason why we need in the proof that every $S_{i}$ is a homothety is that the set of interval projections $I P(K)$ is invariant under the action of $\mathcal{T}$, i.e. if $\Pi_{M} \in I P(K)$ then $\Pi_{T(M)} \in I P(K)$ for every $T \in \mathcal{T}$. Hence via a similar argument to the one in the proof of Theorem 1.3 one can show that instead of assuming that every similarity is a homothety it is enough to assume that $I P(K)$ is invariant under the action of $\mathcal{T}$.

In Theorem 1.3 the assumption, that $K$ is not contained in any line, is essential because a non-degenerate line segment $[x, y]$ on the plane is a 1-set and is the attractor of some SS-IFS that contains only homotheties, but the orthogonal projection onto every line is an interval projection of $[x, y]$. Due to Remark [1.4 the only self similar sets in the plane of Hausdorff dimension 1 that project onto an interval in every direction are line segments. 
In Proposition 2.2 and in Corollary 2.3 we generalize a result of Mattila [11, Propoition 4.2, Corollary 4.3] on the unrectifiability of self-similar sets as we get rid of the 'open set condition' from the assumptions. This generalization will play an important role in the proof of Theorem 1.3 ,

The following result of Farkas [4, Theorem 1.5] implies that if the similarities contain an irrational rotation then $K$ has no interval projection.

Theorem 1.5. Let $\left\{S_{i}\right\}_{i=1}^{m}$ be an SS-IFS in $\mathbb{R}^{2}$ with attractor $K$ and assume that $\mathcal{H}^{1}(K)<\infty$. If $|\mathcal{T}|=\infty$ then $\mathcal{H}^{1}\left(\Pi_{M}(K)\right)=0$ for every line $M$. Hence $K$ has no interval projection.

\subsection{Moment of inertia}

For $\theta \in \mathbb{R}$ let $L_{\theta} \subseteq \mathbb{R}^{2}$ be the line $\{(t \cos \theta, t \sin \theta)\}_{t \in \mathbb{R}}$ and let $P_{\theta}$ be the projection $\Pi_{L_{\theta}}$. In Physics the moment of inertia of a rigid body in $\mathbb{R}^{3}$ with respect to a rotational axis determines the torque needed for a desired angular acceleration about the rotational axis. It is the rotational motion analog of mass for linear motion and is expressed by $\int r^{2} \mathrm{~d} \mu$ where $r$ is the distance of a point from the rotational axis and $\mu$ is the mass distribution of the rigid body. If the body is a solid of revolution about the rotational axis then it is enough to analyse a 2-dimensional cross section of the body through the rotational axis. In that case $\mu$ is a mass distribution in $\mathbb{R}^{2}=\left\{(x, y) \in \mathbb{R}^{2}: x, y \in \mathbb{R}\right\}$ so in the rest of this section $\mu$ is a mass distribution in $\mathbb{R}^{2}$. If the rotational axis is $L_{\frac{\pi}{2}+\theta}$ then the moment of inertia can be expressed by $\int r^{2} \mathrm{~d} \mu=\int\left|P_{\theta}(x, y)\right|^{2} \mathrm{~d} \mu(x, y)$.

The next proposition states that if $\mu$ is a mass distribution in $\mathbb{R}^{2}$ such that the centre of mass is the origin and the moment of inertia with respect to three different rotational axis are the same then they are the same with respect to every rotational axis. This is well-known in mechanics, but for completeness we include the proof.

Proposition 1.6. Let $\mu$ be a finite Borel measure in $\mathbb{R}^{2}=\left\{(x, y) \in \mathbb{R}^{2}: x, y \in \mathbb{R}\right\}$ such that $\int x^{2}+$ $y^{2} \mathrm{~d} \mu(x, y)<\infty$ and

$$
\int x \mathrm{~d} \mu(x, y)=\int y \mathrm{~d} \mu(x, y)=0 .
$$

Assume that there exist $c>0$ and three different angles $\theta, \phi, \psi \in[0, \pi)$ such that

$$
c=\int\left|P_{\theta}(x, y)\right|^{2} \mathrm{~d} \mu(x, y)=\int\left|P_{\phi}(x, y)\right|^{2} \mathrm{~d} \mu(x, y)=\int\left|P_{\psi}(x, y)\right|^{2} \mathrm{~d} \mu(x, y) .
$$

Then $\int x \cdot y \mathrm{~d} \mu(x, y)=0$ and $\int\left|P_{\gamma}(x, y)\right|^{2} \mathrm{~d} \mu(x, y)=c$ for every $\gamma \in \mathbb{R}$.

Proof. For every $\gamma \in \mathbb{R}$

$$
\begin{aligned}
& \int\left|P_{\gamma}(x, y)\right|^{2} \mathrm{~d} \mu(x, y)=\int(x \cos \gamma+y \sin \gamma)^{2} \mathrm{~d} \mu(x, y) \\
& =\cos ^{2} \gamma \int x^{2} \mathrm{~d} \mu(x, y)+\sin ^{2} \gamma \int y^{2} \mathrm{~d} \mu(x, y)+2 \cos \gamma \sin \gamma \int x y \mathrm{~d} \mu(x, y)= \\
& {\left[\begin{array}{ll}
\cos \gamma & \sin \gamma
\end{array}\right]\left[\begin{array}{cc}
\int x^{2} \mathrm{~d} \mu & \int x y \mathrm{~d} \mu \\
\int x y \mathrm{~d} \mu & \int y^{2} \mathrm{~d} \mu
\end{array}\right]\left[\begin{array}{l}
\cos \gamma \\
\sin \gamma
\end{array}\right] .}
\end{aligned}
$$

We can think of the $2 \times 2$ symmetric matrix in the middle as a symmetric bilinear form in the plane. The proposition follows from the fact that a symmetric bilinear form $\beta$ in the plane is uniquely determined by the quantities $\beta(v, v)$ for three pairwise independent vectors $v$.

Remark 1.7. The symmetric bilinear form, appearing in the previous proof, is known in mechanics as the moment of inertia tensor.

For a Borel measure $\mu$ on $\mathbb{R}^{2}$, a subspace $M \subseteq \mathbb{R}^{2}$ and a Borel function $f: \mathbb{R}^{2} \longrightarrow M$ let $f^{*} \mu()=$. $\mu\left(f^{-1}().\right)$ be the image measure in $f\left(\mathbb{R}^{2}\right)$. In the case when $f=P_{\theta}$ we identify $P_{\theta}\left(\mathbb{R}^{2}\right)=L_{\theta}=\{(t \cos \theta, t \sin \theta)\}_{t \in \mathbb{R}}$ with $\mathbb{R}$. 
Corollary 1.8. Let $\mu$ be a finite Borel measure in $\mathbb{R}^{2}=\left\{(x, y) \in \mathbb{R}^{2}: x, y \in \mathbb{R}\right\}$ such that $\int x^{2}+y^{2} \mathrm{~d} \mu(x, y)<$ $\infty$. Assume that there exist three angles $\theta, \phi, \psi \in[0, \pi)$ such that

$$
P_{\theta}^{*} \mu=P_{\phi}^{*} \mu=P_{\psi}^{*} \mu .
$$

Then $\int x \cdot y \mathrm{~d} \mu(x, y)=0$ and there exists $c>0$ such that $\int\left|P_{\gamma}(x, y)\right|^{2} \mathrm{~d} \mu(x, y)=c$ for every $\gamma \in \mathbb{R}$.

If we knew that $\int x \mathrm{~d} \mu(x, y)=\int y \mathrm{~d} \mu(x, y)=0$ then Corollary 1.8 would be an immediate consequence of Proposition [1.6 since $\int\left|P_{\gamma}(x, y)\right|^{2} \mathrm{~d} \mu(x, y)=\int|(x, y)|^{2} \mathrm{~d} P_{\gamma}^{*} \mu(x, y)$ for every $\gamma \in \mathbb{R}$. To see that $\int x \mathrm{~d} \mu(x, y)=\int y \mathrm{~d} \mu(x, y)=0$ we need to show that the centre of mass is the origin. It follows by an easy trigonometric calculation using the trigonometric identity $\frac{\cos \alpha-\cos \beta}{\sin \alpha-\sin \beta}=-\tan \left(\frac{\alpha+\beta}{2}\right)$ and that

$$
\int P_{\theta}(x, y) \mathrm{d} \mu(x, y)=\int P_{\phi}(x, y) \mathrm{d} \mu(x, y)=\int P_{\psi}(x, y) \mathrm{d} \mu(x, y) .
$$

In other words, if the projection of the centre of mass onto three different lines has the same distance from the origin then it has to be the origin. We leave for the reader to check the details.

The following result of Farkas [4, Theorem 1.3] says that if $\mathcal{H}^{1}\left(P_{\theta}(K)\right)>0$ for a self-similar 1-set $K$ then the projection measure is constant times the restriction of Lebesgue measure to the projection.

Theorem 1.9. Let $K \subseteq \mathbb{R}^{2}$ be a self-similar 1-set. If $\mathcal{H}^{1}\left(P_{\theta}(K)\right)>0$ then

$$
P_{\theta}^{*}\left(\left.\mathcal{H}^{1}\right|_{K}\right)=\left.\frac{\mathcal{H}^{1}(K)}{\mathcal{H}^{1}\left(P_{\theta}(K)\right)} \cdot \mathcal{H}^{1}\right|_{P_{\theta(K)}} .
$$

We establish an invariance result of the moment of inertia of self-similar sets with several interval projections. The conclusion is that if we rotate the self-similar set about the centre of mass by an arbitrary angle then the moment of inertia does not change with respect to a fixed axis.

Theorem 1.10. Let $K \subseteq \mathbb{R}^{2}$ be a self-similar 1-set. Assume that there are three different lines through the origin such that the orthogonal projection of $K$ onto them is an interval of length $c$ centered at the origin. Then for $\mu=\left.\mathcal{H}^{1}\right|_{K}$ we have that $\int x \cdot y \mathrm{~d} \mu(x, y)=0$ and $\int\left|P_{\gamma}(x, y)\right|^{2} \mathrm{~d} \mu(x, y)=\mathcal{H}^{1}(K) \cdot c^{2} / 12$ for every $\gamma \in \mathbb{R}$. Furthermore, if $P_{\theta}(K)$ is an interval for some $\theta \in \mathbb{R}$ then $P_{\theta}(K)$ is an interval of length $c$ centered at the origin.

Theorem 1.10 follows from Theorem 1.9, Corollary 1.8 and the fact that $\int_{0}^{a} x^{2} \mathrm{~d} x=a^{3} / 3$.

\section{Rectifiability of self-similar sets}

In this section we generalise a result of Mattila [11, Propoition 4.2, Corollary 4.3] on the rectifiability of self-similar sets as we remove the separation condition from the assumptions. Proof follows Mattila's proof hence we only indicate the differences in the proof.

For $K \subseteq \mathbb{R}^{d}, a \in K, s \in \mathbb{R}$ we denote the $s$-dimensional upper density of $K$ in $a$ by

$$
\Theta^{* s}(K, a)=\limsup _{r \rightarrow 0} \frac{\mathcal{H}^{s}(K \cap B(a, r))}{(2 r)^{s}}
$$

and the $s$-dimensional lower density of $K$ in $a$ by

$$
\Theta_{*}^{s}(K, a)=\liminf _{r \rightarrow 0} \frac{\mathcal{H}^{s}(K \cap B(a, r))}{(2 r)^{s}} .
$$

Definition 2.1. Let $K \subseteq \mathbb{R}^{d}$ and $0 \leq s \leq d$. We say that $K$ is weakly $(s, l)$-tangential at a point $a \in \mathbb{R}^{d}$ if $\Theta^{* s}(K, a)>0$ and there is an affine $l$-plane $M$ such that $a \in M$ and for every $\delta>0$

$$
\liminf _{r \rightarrow 0} r^{-s} \cdot \mathcal{H}^{s}((E \cap B(a, r)) \backslash\{x: \operatorname{dist}(x, M) \leq \delta\})=0 .
$$

Then $M$ is called a weak $(s, l)$-tangent plane of $K$ at $a$. 
Proposition 2.2. Let $K$ be a self-similar set and $s=\operatorname{dim}_{H}(K)$. Suppose that $\mathcal{H}^{s}(K)>0$ and $K$ has a weak tangent plane $M$ at some point $a \in K$. Then $K \subseteq M$.

Proposition 2.2 was proved by Mattila [11. Theorem 4.2] in the case when the 'open set condition' is satisfied. The same proof, that was used by Mattila, can be applied to prove Proposition 2.2 in the general case. The only points where the proof uses the 'open set condition' are that $0<\mathcal{H}^{s}(K)<\infty$ and a result of Hutchinson ([6, Theorem 5.3 (1) (i)]) that states that there exists $\lambda>0$ such that $\Theta_{*}^{s}(K, a) \geq \lambda$ for all $a \in K$. By an application of the implicit methods [2, Thm 3.2] we can deduce that $\mathcal{H}^{s}(K)<\infty$, and by assumption $\mathcal{H}^{s}(K)>0$. To prove, that there exists $\lambda>0$ such that $\Theta_{*}^{s}(K, a) \geq \lambda$ for all $a \in K$, one can follow Hutchinson's proof that only depends on the facts that $0<\mathcal{H}^{s}(K)<\infty$ and if $r_{\min }=\min \left\{r_{i}: i \in \mathcal{I}\right\}$ then $B(a, r)$ contains $K_{\mathbf{i}}$ for some $\mathbf{i} \in \bigcup_{k=1}^{\infty} \mathcal{I}^{k}$ such that $r \cdot r_{\min } \leq r_{\mathbf{i}} \cdot \operatorname{diam}(K) \leq r$ for every small $r$ and $a \in K$. We leave for the reader to check the details.

Corollary 2.3. Let $K$ be a self-similar set, let $s=\operatorname{dim}_{H}(K)$ and $l \in \mathbb{N}, l \geq s$. Then either $K \subseteq M$ for some l-dimensional affine subspace $M$ or $\mathcal{H}^{s}(K \cap \Gamma)=0$ for every l-dimensional $C^{1}$ submanifold $\Gamma$ of $\mathbb{R}^{d}$.

Corollary 2.3 can be deduced from Proposition 2.2 exactly the same way as [11, Corollary 4.3] is deduced from [11, Theorem 4.2].

\section{Isolated interval projections}

This section provides the main tool, Lemma 3.4 to prove that an interval projection is isolated in the set of projections. At the end of the section, Lemma 3.5, we show that the set of interval projections is compact.

For a set $H \subseteq \mathbb{R}^{d}$ we denote the convex hull of $H$ by $\operatorname{Conv}(H)$ and if $F \subseteq \mathbb{R}^{d}$ we denote the distance between $H$ and $F$ by $\operatorname{dist}(H, F)=\inf _{x \in H, y \in F}\|x-y\|$. On the plane the set of all lines through the origin $G_{2,1}$ can be parameterized as $G_{2,1}=\left\{L_{\theta}: \theta \in \mathbb{R} / \pi \mathbb{Z}\right\}$. Let $\prod_{2,1}$ denote the set of all orthogonal projections onto the lines through the origin. Then $\prod_{2,1}=\left\{\Pi_{L}: L \in G_{2,1}\right\}=\left\{P_{\theta}: \theta \in \mathbb{R} / \pi \mathbb{Z}\right\}$ hence $\prod_{2,1}$ inherits a topology and with this topology $\prod_{2,1}$ is compact. For a set $K \subseteq \mathbb{R}^{2}$ we denote the set of all interval projections of $K$ by $I P(K)$.

Lemma 3.1. Let $K \subseteq \mathbb{R}^{2}$ be a compact set, $A \subseteq K$ be a compact subset such that $I P(K) \subseteq I P(A)$ and let $L^{1}$ and $L^{2}$ be lines parallel to the $y$-axis such that $A$ stays between $L^{1}$ and $L^{2}$, both $L^{1}$ and $L^{2}$ intersect $A$ and the $x$-coordinate of the points of $L^{1}$ is smaller then the $x$-coordinate of the points of $L^{2}$. Let $B \subseteq K$ be a compact subset such that for every $P_{\theta} \in I P(K)$ we have that $\mathcal{H}^{1}\left(P_{\theta}(A) \cap P_{\theta}(B)\right)=0$ and for every open set $U$ that intersects $B$ and for every $P_{\theta} \in I P(K)$ we have that $0<\mathcal{H}^{1}\left(P_{\theta}(B \cap U)\right)$.

i) If either $B$ contains a point $z$ of $L^{1}$ such that there exists $w \in A \cap L^{1}$ with $y$-coordinate smaller than the $y$-coordinate of $z$ or $B$ contains a point $z$ of $L^{2}$ such that there exists $w \in A \cap L^{2}$ with $y$-coordinate greater than the $y$-coordinate of $z$, then there exists $\delta>0$ such that for every $0<\theta<\delta$ we have that $P_{\theta} \notin I P(K)$.

ii) If either $B$ contains a point $z$ of $L^{1}$ such that there exists $w \in A \cap L^{1}$ with $y$-coordinate greater than the $y$-coordinate of $z$ or $B$ contains a point $z$ of $L^{2}$ such that there exists $w \in A \cap L^{2}$ with $y$-coordinate smaller than the $y$-coordinate of $z$, then there exists $\delta>0$ such that for every $-\delta<\theta<0$ we have that $P_{\theta} \notin I P(K)$.

Proof. i) Assume that the case $w, z \in L^{1}$ holds (the proof of the other case, when $w, z \in L^{2}$ holds, goes similarly to the proof of this case or alternatively can be deduced from this case by rotating everything around the origin by $\pi)$. Let $w_{2} \in A \cap L^{2}$ and let $\delta \in(0, \pi)$ be such that if we draw a line $L^{3}$ through $z$ and $w_{2}$ then $L^{3}$ is parallel to $L_{\frac{\pi}{2}+\delta}$. We claim that $P_{\theta} \notin I P(K)$ for every $0<\theta<\delta$. Assume for a contradiction that $P_{\theta} \in I P(K)$ for some $0<\theta<\delta$. Let $L^{4}$ be the line through $z$ such that $L^{4}$ is parallel to $L_{\frac{\pi}{2}+\theta}$. Then $L^{4}$ lies strictly between the points $w$ and $w_{2}$ hence $P_{\theta}(z)$ is in the interior of the interval Conv $\left(\left\{P_{\theta}(w), P_{\theta}\left(w_{2}\right)\right\}\right)$ (see Figure 1). Since $w, w_{2} \in A$ and by assumption $P_{\theta} \in I P(K) \subseteq I P(A)$ it follows that $P_{\theta}(A)$ is an interval and $\operatorname{Conv}\left(\left\{P_{\theta}(w), P_{\theta}\left(w_{2}\right)\right\}\right) \subseteq P_{\theta}(A)$. Thus $P_{\theta}(z)$ is in the interior of the interval $P_{\theta}(A)$. Let $U$ be an open neighborhood of $z$ such that $P_{\theta}(U) \subseteq P_{\theta}(A)$. Then $P_{\theta}(B \cap U) \subseteq P_{\theta}(A) \cap P_{\theta}(B)$ and by assumption $0<\mathcal{H}^{1}\left(P_{\theta}(B \cap U)\right)$. Hence $0<\mathcal{H}^{1}\left(P_{\theta}(A) \cap P_{\theta}(B)\right)$ but this contradicts the assumption of the lemma.

The proof of ii) goes similarly to the proof of $i$ ) or alternatively ii) can be deduced from i) by reflecting everything in the $y$-axis. 


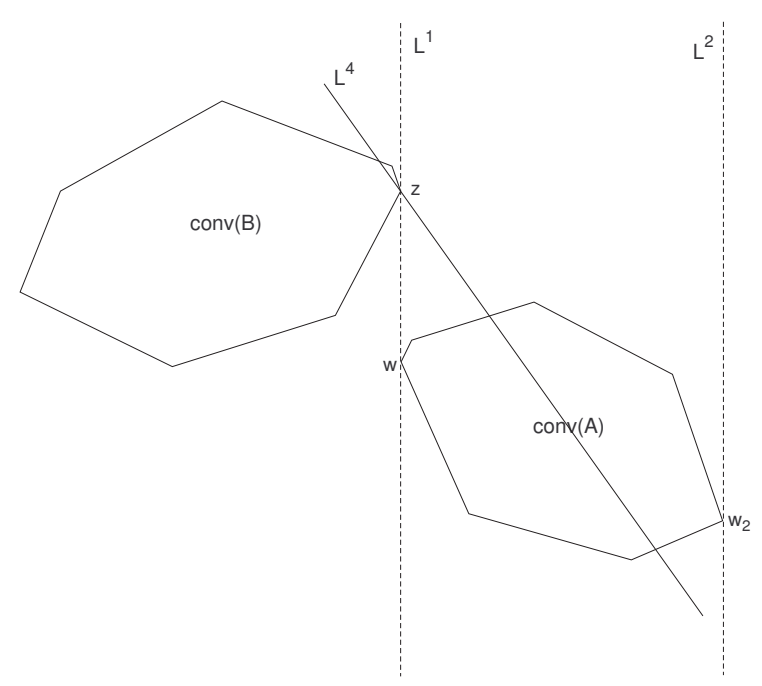

Figure 1.

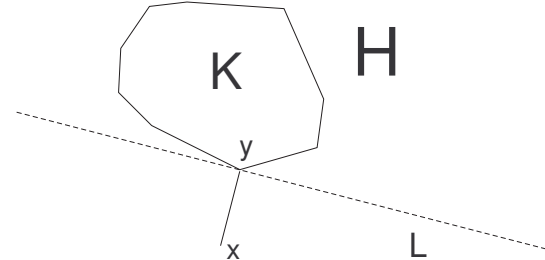

Figure 2.

Lemma 3.2. Let $K \subseteq \mathbb{R}^{2}$ be a convex, compact set and let $x \in \mathbb{R}^{2} \backslash K$ and $y \in K$ such that $\operatorname{dist}(\{x\}, K)=$ $\|x-y\|$. Let $L$ be the line through $y$ that is perpendicular to the line segment $[x, y]$ and let $H$ be the closed half-plane that is bordered by $L$ and $x \notin H$. Then $K \subseteq H$.

The proof is trivial geometric argument, that if $z \in K \backslash H$ then there is a closer point to $x$ than $y$ on the line segment $[y, z]$. See Figure 2 .

Lemma 3.3. Let $K \subseteq \mathbb{R}^{2}$ be a compact set. Let $A, B \subseteq K$ be compact subsets such that $C$ onv $(A) \cap$ $\operatorname{Conv}(B)=\emptyset, P_{0} \in I \bar{P}(A), P_{0} \in I P(B), \operatorname{diam}\left(P_{0}(A)\right)>0, \operatorname{diam}\left(P_{0}(B)\right)>0$ and $\left|P_{0}(A) \cap P_{0}(B)\right|=1$. Then $P_{0} \in I P(A \cup B)$. Assume that $P_{0}(K \backslash(A \cup B))$ and the interior of the interval $P_{0}(A \cup B)$ are disjoint. Let $L$ be the line through $P_{0}(A) \cap P_{0}(B)$ that is parallel to the $y$-axis.

i) If either $A$ is to the right of $L$ and there exist $w \in A \cap L$ and $z \in B \cap L$ such that the $y$-coordinate of $w$ is smaller than the $y$-coordinate of $z$ or $A$ is to the left of $L$ and there exist $w \in A \cap L$ and $z \in B \cap L$ such that the $y$-coordinate of $w$ is greater than the $y$-coordinate of $z$, then there exists $\delta>0$ such that for every $-\delta<\theta<0$ we have that $P_{\theta} \notin I P(K)$.

ii) If either $A$ is to the right of $L$ and there exist $w \in A \cap L$ and $z \in B \cap L$ such that $y$-coordinate of $w$ is greater than the $y$-coordinate of $z$ or $A$ is to the left of $L$ and there exist $w \in A \cap L$ and $z \in B \cap L$ such that the $y$-coordinate of $w$ is smaller than the $y$-coordinate of $z$, then there exists $\delta>0$ such that for every $0<\theta<\delta$ we have that $P_{\theta} \notin I P(K)$.

Proof. i) Assume that the case, $A$ is to the right of $L$, holds (the proof of the other case goes similarly to the proof of this case or alternatively can be deduced from this case by rotating everything around the origin by $\pi)$. Since $P_{0}(A)$ and $P_{0}(B)$ are intervals that intersect we have that $P_{0}(A \cup B)$ is an in interval, so $P_{0} \in I P(A \cup B)$.

Since $A$ and $B$ are compact $\operatorname{Conv}(A)$ and $\operatorname{Conv}(B)$ are compact sets. By assumption $\operatorname{Conv}(A) \cap$ $\operatorname{Conv}(B)=\emptyset$. Hence $\alpha=\operatorname{dist}(\operatorname{Conv}(A), \operatorname{Conv}(B))>0$. By compactness there exist $g \in B, h \in A$ such that $\|h-g\|=\alpha$. Let $L^{5}$ be the perpendicular bisector of the line segment $[g, h]$ and let $\delta_{1} \in(0, \pi)$ such that $L_{\frac{\pi}{2}-\delta_{1}}$ is parallel to $L^{5}$ (note that we can choose such $\delta_{1} \in(0, \pi)$ since $L^{5}$ is not parallel to the $y$-axis as the convex sets $\operatorname{Conv}(A)$ and $\operatorname{Conv}(B)$ both intersect $L$ but stay on different sides of $L)$. So $\left|L \cap L^{5}\right|=1$ and let $p \in \mathbb{R}^{2}$ be the point of intersection $L \cap L^{5}$. By Lemma 3.2 $\operatorname{Conv}(A)$ stays below $L^{5}$ and $C o n v(B)$ stays above $L^{5}$.

Since $\left|P_{0}(A) \cap P_{0}(B)\right|=1$ the compact intervals $P_{0}(A)$ and $P_{0}(B)$ intersect in one point and $P_{0}(A)$ is to the right of the intersection. Let $a<b<c$ on the line $\mathbb{R} \times\{0\}=\mathbb{R}$ such that $P_{0}(B)=[a, b]$ and $P_{0}(A)=[b, c]$ and hence $P_{0}(L)=\{b\}$. Since $K$ is compact there exists $R>0$ such that $K$ is contained in the square $[-R, R] \times[-R, R]$. In particular, $A \cup B$ is contained in the rectangle $[a, c] \times[-R, R]$. Let $\delta_{2} \in(0, \pi)$ be such that for every $-\delta_{2}<\theta<0$ the line through $p$, that is parallel to $L_{\frac{\pi}{2}}+\theta$, intersects both $[a, b] \times\{-R\}$ and $[b, c] \times\{R\}$.

Let $\delta=\min \left\{\delta_{1}, \delta_{2}\right\}$. We claim that $P_{\theta} \notin I P(K)$ for every $-\delta<\theta<0$. Let $-\delta<\theta<0$ be arbitrary and let $L^{6}$ be the line through $p$ that is parallel to $L_{\frac{\pi}{2}+\theta}$. By the choice of $\delta_{1}$ the line $L^{6}$ avoids $C o n v(A)$ 
and $\operatorname{Conv}(B)$ and hence avoids $A$ and $B$ (see Figure 3). By the assumption, that $P_{0}(K \backslash(A \cup B)$ ) and the interior of the interval $P_{0}(A \cup B)$ are disjoint, it follows that $K \backslash(A \cup B)$ does not intersect $(a, c) \times \mathbb{R}$ but by the choice of $\delta_{2}$ we have that $L^{6}$ intersects $[-R, R] \times[-R, R]$ only inside $(a, c) \times \mathbb{R}$ (see Figure 4 ). Hence by the choice of $R$ we have that $K \backslash(A \cup B)$ does not intersect $L^{6}$. So $L^{6}$ does not intersect $A, B$ and $K \backslash(A \cup B)$ thus $L^{6}$ does not intersect $K$. Hence $P_{\theta}(p) \notin P_{\theta}(K)$. But since $C o n v(A)$ stays below $L^{6}$ and $\operatorname{Conv}(B)$ stays above $L^{6}$ and so $A$ stays below $L^{6}$ and $B$ stays above $L^{6}$ we have that $P_{\theta}(p) \in C o n v\left(P_{\theta}(K)\right)$. Thus $P_{\theta}(K)$ is not an interval.

The proof of $i$ ) goes similarly to the proof of $i$ ) or alternatively ii) can be deduced from $i$ ) by reflecting everything in the $y$-axis.

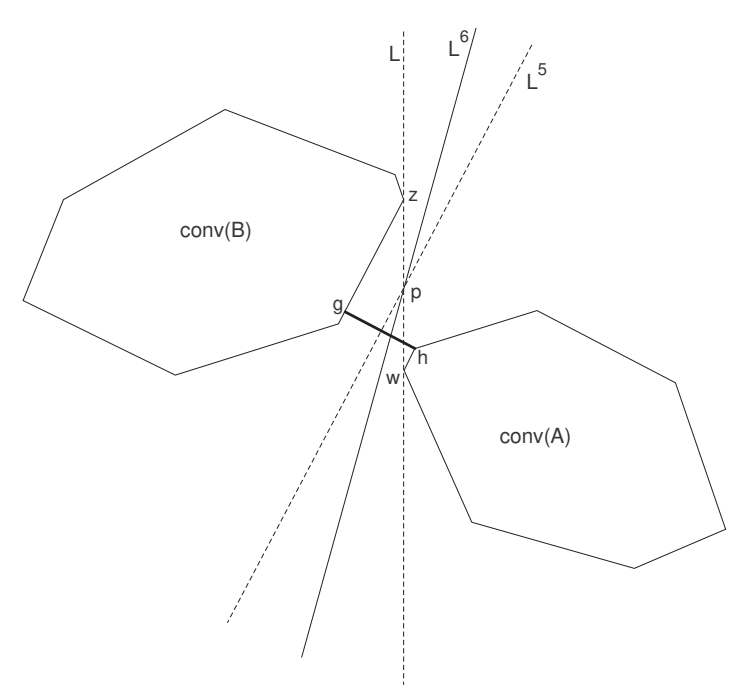

Figure 3.

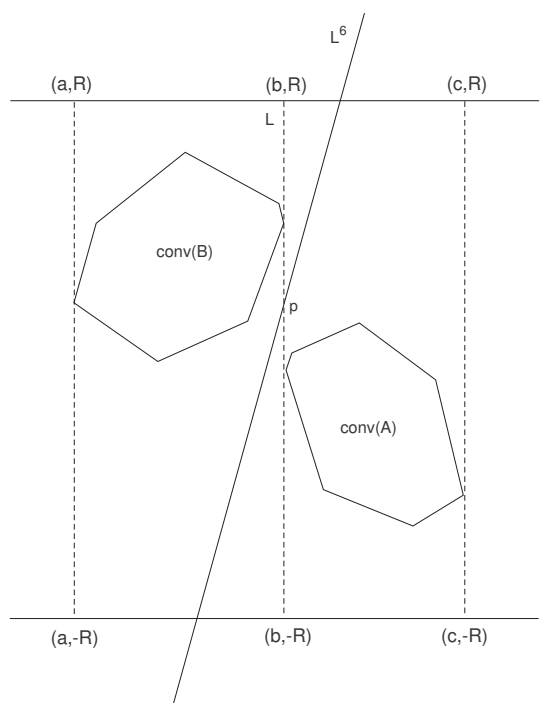

Figure 4.

Lemma 3.4. Let $K \subseteq \mathbb{R}^{2}$ be a compact set. Let $A \subseteq K$ be a compact subset such that $I P(K) \subseteq I P(A)$, $P_{0} \in I P(A)$ and $\operatorname{diam}\left(P_{0}(A)\right)>0$. Let $B \subseteq K$ be a compact subset such that for every $P_{\theta} \in I P(K)$ we have that $\mathcal{H}^{1}\left(P_{\theta}(A) \cap P_{\theta}(B)\right)=0$, for every open set $U$ that intersects $B$ and for every $P_{\theta} \in I P(K)$ we have that $0<\mathcal{H}^{1}\left(P_{\theta}(B \cap U)\right), P_{0} \in I P(B), \operatorname{diam}\left(P_{0}(B)\right)>0,\left|P_{0}(A) \cap P_{0}(B)\right|=1$ and $A \cap B=\emptyset$. Then $P_{0} \in I P(A \cup B)$. Assume that $P_{0}(K \backslash(A \cup B))$ and the interior of the interval $P_{0}(A \cup B)$ are disjoint. Then there exists $\delta>0$ such that for every $\theta \in(-\delta, \delta) \backslash\{0\}$ we have that $P_{\theta} \notin I P(K)$.

Proof. Since $P_{0}(A)$ and $P_{0}(B)$ are intervals that intersect it follows that $P_{0}(A \cup B)$ is an in interval and so $P_{0} \in I P(A \cup B)$. Let $L$ be the line through $P_{0}(A) \cap P_{0}(B)$ that is parallel to the $y$-axis. Assume that the case that $A$ is to the right of $L$ holds (the proof of the other case goes similarly to the proof of this case or alternatively can be deduced from this case by rotating everything around the origin by $\pi)$. Let $E_{A}=\{y \in \mathbb{R}: \exists x \in \mathbb{R},(x, y) \in A \cap L\}$ be the set of the $y$-coordinates of the points of $A \cap L$ and let $E_{B}=\{y \in \mathbb{R}: \exists x \in \mathbb{R},(x, y) \in B \cap L\}$ be the set of the $y$-coordinates of the points of $B \cap L$. Let $I_{A}=\operatorname{Conv}\left(E_{A}\right)$ and $I_{B}=\operatorname{Conv}\left(E_{B}\right)$. Since $A \cap B=\emptyset$ we have that $E_{A}$ and $E_{B}$ are disjoint compact sets. Hence either $I_{A}$ and $I_{B}$ are disjoint intervals or $I_{A}$ and $I_{B}$ are overlapping intervals, i.e. the interior of $I_{A} \cap I_{B}$ is nonempty. We will consider these two cases in the proof.

Case 1: $I_{A}$ and $I_{B}$ are disjoint intervals

Case 2: $I_{A}$ and $I_{B}$ are overlapping intervals

Case 1: Assume that the case $\sup I_{A}<\inf I_{B}$ holds (the proof of the other case, $\inf I_{A}>\sup I_{B}$, goes similarly to the proof of this case or alternatively can be deduced from this case by reflecting everything in the $x$-axis). Since $\sup I_{A}<\inf I_{B}$ with the notation $L=L^{1}$ we have that $B$ contains a point $z$ of $L^{1}$ such that there exists $w \in A \cap L^{1}$ with $y$-coordinate smaller than the $y$-coordinate of $z$. So the conditions of Lemma $3.1 i$ ) are satisfied. Since $P_{0}(A)$ and $P_{0}(B)$ are non-overlapping intervals and $I_{A}$ and $I_{B}$ are disjoint intervals it follows that $\operatorname{Conv}(A) \cap \operatorname{Conv}(B)=\emptyset$. Again since $\sup I_{A}<\inf I_{B}$ it follows that there exist a $w \in A \cap L$ and $z \in B \cap L$ such that $y$-coordinate of $w$ is smaller than the $y$-coordinate of $z$. We assumed that $A$ is to the right of $L$. So the conditions of Lemma $3.3 i$ ) are satisfied. So the conditions of both 
Lemma 3.1 i) and Lemma $3.3 i$ ) are satisfied. Hence there exists $\delta>0$ such that $P_{\theta} \notin I P(K)$ for every $\theta \in(-\delta, \delta) \backslash\{0\}$.

Case 2: Since $I_{A}$ and $I_{B}$ are overlapping intervals with the notation $L=L^{1}$ we have that $B$ contains a point $z_{1}$ of $L^{1}$ such that there exists $w_{1} \in A \cap L^{1}$ with $y$-coordinate smaller than the $y$-coordinate of $z_{1}$, and that $B$ contains a point $z_{2}$ of $L^{1}$ such that there exists $w_{2} \in A \cap L^{1}$ with $y$-coordinate greater than the $y$-coordinate of $z_{2}$. So the conditions of Lemma 3.1 i) are satisfied with $z=z_{1}$ and the conditions of Lemma 3.1 ii) are satisfied with $z=z_{2}$. Hence there exists $\delta>0$ such that $P_{\theta} \notin I P(K)$ for every $\theta \in(-\delta, \delta) \backslash\{0\}$.

Lemma 3.5. Let $K \subseteq \mathbb{R}^{2}$ be compact. Then $I P(K)$ is compact.

Proof. Since $\prod_{2,1}$ is compact it is enough to show that $I P(K)$ is closed i.e. $\prod_{2,1} \backslash I P(K)$ is open. Let $P_{\theta} \in \prod_{2,1} \backslash I P(K)$. We need to show that a neighborhood of $P_{\theta}$ is contained in $\prod_{2,1} \backslash I P(K)$. Without the loss of generality we can assume that $\theta=0$ otherwise we can rotate everything around the origin by $-\theta$. Since $P_{0}$ is not an interval projection of $K$ it follows that $P_{0}(K)$ is not an interval. Let $x \in \operatorname{Conv}\left(P_{0}(K)\right) \backslash P_{0}(K)$. Since $K$ is compact it follows that $P_{0}(K)$ is compact as well and hence there exists $r>0$ such that the interval $[x-r, x+r]$ is contained in $\operatorname{Conv}\left(P_{0}(K)\right) \backslash P_{0}(K)$. Since $K$ is compact there exists $R>0$ such that $K$ is contained in the square $[-R, R] \times[-R, R]$. Let $y=\inf P_{0}(K)$ and $z=\sup P_{0}(K)$. Then $K$ is contained in $[y, x-r) \times[-R, R] \cup(x+r, z] \times[-R, R]$ and both components intersect $K$. Let $\delta>0$ be such that the line, that connects the points $(x-r, R)$ and $(x+r,-R)$, is parallel to $L_{\frac{\pi}{2}+\delta}$. Then for every $\varphi \in(-\delta, \delta)$ the line, that goes through the point $(x, 0)$ and is parallel to $L_{\frac{\pi}{2}+\varphi}$, does not intersect $K$ and separates $K$ into two non-empty part. Hence $P_{\varphi}(K)$ is not an interval for every $\varphi \in(-\delta, \delta)$.

\section{Interval projections of self-similar sets}

In this section we prove the main results of the paper, Theorem 1.2 and Theorem 1.3 Since $I P(K)$ is compact by Lemma 3.5 it is enough to prove that every element of $I P(K)$ is isolated. We proceed by finding subsets $A, B \subseteq K$ that satisfies the assumption of Lemma 3.4 .

In this section let $\left\{S_{i}\right\}_{i=1}^{m}$ be a SS-IFS in $\mathbb{R}^{2}$ with attractor $K$ and $S_{i}$ be decomposed as in (2) and we denote $\max \left\{r_{i}\right\}_{i=1}^{m}<1$ by $r_{\text {max }}$. We further assume that $\operatorname{dim}_{H}(K)=1$.

Lemma 4.1. If $\mathcal{H}^{1}\left(P_{\theta}(K)\right)>0$ then for every open set $U$ that intersects $K$ we have that $0<\mathcal{H}^{1}\left(P_{\theta}(K \cap U)\right)$.

Proof. Let $P_{\theta} \in \prod_{2,1}$ such that $\mathcal{H}^{1}\left(P_{\theta}(K)\right)>0$, let $U$ be an open set that intersects $K$ and $x \in K \cap U$. Then there exists a ball $B$, centered at $x$ with radius $r_{U}>0$, such that $B \subseteq U$. There exists a cylinder set $K_{\mathbf{i}} \subseteq K \cap U$ for large enough $k$ and for some $\mathbf{i} \in \mathcal{I}^{k}$. By [4, Theorem 1.3] we have that

$$
\mathcal{H}^{1}\left(P_{\theta}(K \cap U)\right) \geq \mathcal{H}^{1}\left(P_{\theta}\left(K_{\mathbf{i}}\right)\right)=\frac{\mathcal{H}^{1}\left(K_{\mathbf{i}}\right)}{\mathcal{H}^{1}(K)} \cdot \mathcal{H}^{1}\left(P_{\theta}(K)\right)=r_{\mathbf{i}}^{k} \cdot \mathcal{H}^{1}\left(P_{\theta}(K)\right)>0 .
$$

Lemma 4.2. Let $A, B \subseteq K$ be disjoint compact subsets. Then $\mathcal{H}^{1}\left(P_{\theta}(A) \cap P_{\theta}(B)\right)=0$ for every $P_{\theta} \in \prod_{2,1}$.

For details of the proof see [4, Corolllary 1.4].

Proof of Theorem 1.2. We need to show that $I P(K)$ is finite. Since $K$ is compact, $I P(K)$ is a compact subset of $\prod_{2,1}$ by Lemma 3.5. Thus it is enough to show that every $P_{\theta} \in I P(K)$ is isolated in $I P(K)$. If $I P(K)=\emptyset$ the proof is trivial, so we assume that $I P(K) \neq \emptyset$. Hence by Theorem [1.5 we have that $|\mathcal{T}|<\infty$. If $K$ is contained in a line, that is parallel to $L_{\frac{\pi}{2}+\gamma}$ for some $\gamma \in[0, \pi)$, then $|I P(K)|=1$ because $\left\{S_{i}\right\}_{i=1}^{m}$ satisfies the SSC and so the only interval projection of $K$ is $P_{\gamma}$. So we assume that $q=|\mathcal{T}|<\infty$ and $K$ is not contained in any line.

Let $P_{\theta} \in I P(K)$ be arbitrary. We need to show that there exists $\delta>0$ such that $P_{\theta+\varphi} \notin I P(K)$ for every $\varphi \in(-\delta, \delta) \backslash\{0\}$. Without the loss of generality we can assume that $\theta=0$ otherwise we can rotate everything around the origin by $-\theta$. Let $i_{1} \in \mathcal{I}$ be arbitrary and $\mathbf{i}=\left(i_{1}, \ldots, i_{1}\right) \in \mathcal{I}^{2 \cdot q}$. Then $T_{\mathbf{i}}=T_{i_{1}}^{2 \cdot q}$ is the the identity map because $q=|\mathcal{T}|<\infty$. So $K_{\mathbf{i}}=r_{\mathbf{i}} \cdot K+t_{\mathbf{i}}$ and hence $\operatorname{IP}\left(K_{\mathbf{i}}\right)=I P(K)$. Since $P_{0} \in I P(K)$ let

$$
P_{0}(K)=[a, b] \subseteq \mathbb{R} \times\{0\}=\mathbb{R}
$$


and

$$
P_{0}\left(K_{\mathbf{i}}\right)=[c, d] \subseteq \mathbb{R} \times\{0\}=\mathbb{R}
$$

and both $[a, b]$ and $[c, d]$ are intervals of positive length because $K$ is not contained in any line. Then either $[a, c]$ or $[d, b]$ is an interval of positive length. Let this interval of positive length be $J$, let $B=$ $\left(P_{0}^{-1}(J) \cap K\right) \backslash K_{\mathbf{i}}$ and $A=K_{\mathbf{i}}$. Then $B$ is compact by that $\left\{S_{i}\right\}_{i=1}^{m}$ satisfies the SSC. So $P_{0} \in I P(K)=$ $I P(A), P_{0} \in I P(B), A$ and $B$ are disjoint compact subsets of $K,\left|P_{0}(A) \cap P_{0}(B)\right|=1, \operatorname{diam}\left(P_{0}(A)\right)>0$, $\operatorname{diam}\left(P_{0}(B)\right)>0, P_{0} \in I P(A \cup B), P_{0}(K \backslash(A \cup B))$ and the interior of the interval $P_{0}(A \cup B)$ are disjoint and by Lemma 4.2 for every $P_{\theta} \in \prod_{2,1}$ we have that $\mathcal{H}^{1}\left(P_{\theta}(A) \cap P_{\theta}(B)\right)=0$. By Lemma 4.1 for every open set $U$ that intersects $B$ and for every $P_{\theta} \in I P(K)$ we have that $0<\mathcal{H}^{1}\left(P_{\theta}(B \cap U)\right)$. So all the conditions of Lemma 3.4 are satisfied for $A, B \subseteq K$. Hence there exists $\delta>0$ such that $P_{0+\varphi} \notin I P(K)$ for every $\varphi \in(-\delta, \delta) \backslash\{0\}$. So $P_{0} \in I P(K)$ is isolated in $I P(K)$.

Lemma 4.3. Let $K \subseteq \mathbb{R}^{2}$ be such that $\mathcal{H}^{1}(K)<\infty$. If there exists a non-degenerate continuous curve $\Gamma_{0}$ such that $\Gamma_{0} \subseteq K$ then there exists a 1-dimensional $C^{1}$ submanifold $\Gamma$ of $\mathbb{R}^{2}$ such that $\mathcal{H}^{1}(K \cap \Gamma)>0$.

Proof. If $x, y \in \Gamma_{0}, x \neq y$ then $P_{\theta}(K)$ is an interval of positive length for each $\theta$ such that $L_{\theta}$ is not perpendicular to the line segment $[x, y]$. So the statement follows from [9, Theorem 18.1 (2)].

Proof of Theorem 1.3. As in the proof of Theorem 1.2 it is enough to show that for $P_{\theta} \in I P(K)$ there exists $\delta>0$ such that $P_{\theta+\varphi} \notin I P(K)$ for every $\varphi \in(-\delta, \delta) \backslash\{0\}$ and without the loss of generality we can assume that $\theta=0$. We proceed by showing that there are sets $A, B \subseteq K$ that satisfies the assumptions of Lemma 3.4 otherwise $K$ would contain a continuous curve which would contradict with the unrectifiability of $K$.

Since each $S_{i}$ is a homothety for all $i \in \mathcal{I}$ it follows that $\operatorname{IP}\left(K_{\mathbf{i}}\right)=\operatorname{IP}\left(S_{\mathbf{i}}(K)\right)=I P(K)$ for all $k \in \mathbb{N}$ and $\mathbf{i} \in \mathcal{I}^{k}$. Since $P_{0} \in I P(K)$ let

$$
P_{0}(K)=[a, b] \subseteq \mathbb{R} \times\{0\}=\mathbb{R} .
$$

So $[a, b]$ is an interval of positive length because $K$ is not contained in any line.

We claim that there is a unique $w \in K$ such that $P_{0}(w)=a$. Otherwise, assume for a contradiction that $w^{\prime}$ is another point of $K$ such that $P_{0}\left(w^{\prime}\right)=a$. Let $r_{d}=\left\|w-w^{\prime}\right\|$ and $k \in \mathbb{N}$ such that $r_{\text {max }}^{k} \cdot \operatorname{diam}(K)<\frac{r_{d}}{2}$. Then $\operatorname{diam}\left(K_{\mathbf{i}}\right)<r_{\text {max }}^{k} \cdot \operatorname{diam}(K)<\frac{r_{d}}{2}$ for every $\mathbf{i} \in \mathcal{I}^{k}$. There exist $\mathbf{i}, \mathbf{j} \in \mathcal{I}^{k}$ such that $w \in K_{\mathbf{i}}$ and $w^{\prime} \in K_{\mathbf{j}}$. Since $\operatorname{diam}\left(K_{\mathbf{i}}\right)<\frac{r_{d}}{2}, w \in K_{\mathbf{i}}$ and $\operatorname{diam}\left(K_{\mathbf{j}}\right)<\frac{r_{d}}{2}, w^{\prime} \in K_{\mathbf{j}}$ it follows that $K_{\mathbf{i}}$ and $K_{\mathbf{j}}$ are disjoint. Since $P_{0} \in I P\left(K_{\mathbf{i}}\right)$ and $P_{0} \in I P\left(K_{\mathbf{j}}\right)$ we have that for some $c_{1}, c_{2}>a$ the projections $P_{0}\left(K_{\mathbf{i}}\right)=\left[a, c_{1}\right]$ and $P_{0}\left(K_{\mathbf{j}}\right)=\left[a, c_{2}\right]$ are overlapping intervals. On the other hand, this contradicts with Lemma 4.2. So there is a unique $w \in K$ such that $P_{0}(w)=a$. Similarly there is a unique $z \in K$ such that $P_{0}(z)=b$.

So $P_{0}\left(K_{\mathbf{i}}\right)$ is an interval and for all $\mathbf{i} \in \mathcal{I}^{k}$ and for both endpoints of the interval $P_{0}\left(K_{\mathbf{i}}\right)$ there is a unique point of $K_{\mathbf{i}}$ that projects onto that endpoint of the interval $P_{0}\left(K_{\mathbf{i}}\right)$ because each $S_{\mathbf{i}}$ is a homothety. We say that a cylinder set, $K_{\mathbf{i}}$ for some $\mathbf{i} \in \mathcal{I}^{k}$, is a fitting piece if there exist unique $w_{\mathbf{i}}, z_{\mathbf{i}} \in K$ such that $P_{0}\left(w_{\mathbf{i}}\right)$ and $P_{0}\left(z_{\mathbf{i}}\right)$ are the two endpoints of the interval $P_{0}\left(K_{\mathbf{i}}\right)$. We claim that there exists $\mathbf{i} \in \mathcal{I}^{k}$ for some $k \in \mathbb{N}$ such that $K_{\mathbf{i}}$ is not a fitting piece. Assume for a contradiction that $K_{\mathbf{i}}$ is a fitting piece for each $k \in \mathbb{N}, \mathbf{i} \in \mathcal{I}^{k}$ and without the loss of generality we can assume that the $x$-coordinate of $w_{\mathbf{i}}$ is smaller than the $x$-coordinate of $z_{\mathbf{i}}$. Let $a=x_{0, k}<x_{1, k}<\ldots<x_{n_{k}, k}=b$ such that $\left\{x_{j, k}: j \in\left\{0,1, \ldots, n_{k}\right\}\right\}=$ $\left\{P_{0}\left(w_{\mathbf{i}}\right): \mathbf{i} \in \mathcal{I}^{k}\right\} \bigcup\left\{P_{0}\left(z_{\mathbf{i}}\right): \mathbf{i} \in \mathcal{I}^{k}\right\}$. For $k \in \mathbb{N}$ let $f_{k}:[a, b] \longrightarrow \mathbb{R}$ be the continuous function such that $w_{\mathbf{i}}, z_{\mathbf{i}} \in \operatorname{graph}\left(f_{k}\right)$ for each $\mathbf{i} \in \mathcal{I}^{k}$ and the restriction of $f_{k}$ to the interval $\left[x_{j, k}, x_{j+1, k}\right]$ is linear. If $P_{0}\left(w_{\mathbf{j}}\right) \in P_{0}\left(K_{\mathbf{i}}\right)$ for some $\mathbf{i}, \mathbf{j} \in \mathcal{I}^{k}$ then $w_{\mathbf{j}} \in K_{\mathbf{i}}$ because $K_{\mathbf{j}}$ is a fitting piece. Thus if $P_{0}\left(K_{\mathbf{i}}\right) \cap$ $P_{0}\left(K_{\mathbf{j}}\right) \neq \emptyset$ then the $2 \cdot r_{\text {max }}^{k} \cdot \operatorname{diam}(K)$-neighbourhood of $w_{\mathbf{i}}$ contains $K_{\mathbf{j}}$ and so also contains $f_{k}\left(P_{0}\left(K_{\mathbf{i}}\right)\right)$. Hence $\left|f_{k}(x)-f_{k+l}(x)\right| \leq 4 \cdot r_{\max }^{k} \cdot \operatorname{diam}(K)$ for all $x \in[a, b]$ and $l \in \mathbb{N}$. So the sequence $\left(f_{k}\right)_{=1}^{\infty}$ is uniformly convergent and $f(x):=\lim _{k \rightarrow \infty} f_{k}(x)$ is a continuous function. Then $g r a p h(f)$ is a non-degenerate continuous curve, that is contained in $K$. Hence by Lemma 4.3 there exists a 1-dimensional $C^{1}$ submanifold $\Gamma$ of $\mathbb{R}^{2}$ such that $\mathcal{H}^{1}(K \cap \Gamma)>0$. Thus $K$ is contained in a line by Corollary 2.3 but this contradicts with the assumption of the theorem. So there exists $\mathbf{i} \in \mathcal{I}^{k}$ for some $k \in \mathbb{N}$ such that $K_{\mathbf{i}}$ is not a fitting piece.

Let $K_{\mathbf{i}}$ be a non-fitting piece for some $\mathbf{i} \in \mathcal{I}^{k}$. Then there exists $x \in K$ such that $x \notin K_{\mathbf{i}}$ and $P(x) \in P_{0}\left(K_{\mathbf{i}}\right)$. Because $K_{\mathbf{i}}$ is compact and $x \notin K_{\mathbf{i}}$ it follows that $\operatorname{dist}\left(x, K_{\mathbf{i}}\right)>0$. Let $k_{2} \in \mathbb{N}$ such that $r_{\text {max }}^{k_{2}} \cdot \operatorname{diam}(K)<\operatorname{dist}\left(x, K_{\mathbf{i}}\right)$. There exists $\mathbf{j} \in \mathcal{I}^{k_{2}}$ such that $x \in K_{\mathbf{j}}$. It follows that $K_{\mathbf{i}} \cap K_{\mathbf{j}}=\emptyset$, $P_{0}\left(K_{\mathbf{i}}\right) \cap P_{0}\left(K_{\mathbf{j}}\right) \neq \emptyset$ but by Lemma $4.2\left|P_{0}\left(K_{\mathbf{i}}\right) \cap P_{0}\left(K_{\mathbf{j}}\right)\right|=1$. Let $A=K_{\mathbf{i}}$ and $B=K_{\mathbf{j}}$. By checking, similarly as we did it in the proof of Theorem 1.2, that all the conditions of Lemma 3.4 are satisfied for $A, B \subseteq K$ it follows that there exists $\delta>0$ such that $P_{0+\varphi} \notin I P(K)$ for every $\varphi \in(-\delta, \delta) \backslash\{0\}$. 
Remark 4.4. Let $K \subseteq \mathbb{R}^{d}$. We can define interval projections in higher dimension such that we call $\Pi_{M}$ an interval projection of $K$ for a line $M \subseteq \mathbb{R}^{d}$ through the origin, if $\Pi_{M}(K)$ is an interval with the $M=\mathbb{R}$ identification. For a set $K \subseteq \mathbb{R}^{d}$ we denote the set of all interval projections of $K$ by $\operatorname{IP}(K)$. We can generalize Theorem 1.3 as follows:

Let $\left\{S_{i}\right\}_{i=1}^{m}$ be an SS-IFS in $\mathbb{R}^{d}(d \geq 2)$ with attractor $K$ such that each $S_{i}$ are homotheties and $K$ is a 1-set that is not contained in any affine hyperplane. Then there are at most finitely many lines through the origin, such that the orthogonal projection onto them are interval projections of $K$.

The assumption, that $K$ is not contained in any affine hyperplane, is necessary. Let $K$ be a set contained in a hyperplane $H$, let $v$ be the normal vector of $H$, let $\Pi_{M}$ be an interval projection of $K$ for some line $M \subseteq H$, and let $x$ be a non-zero vector in $M$. Let $M_{n}=\left\{\lambda \cdot\left(x+\frac{1}{n} \cdot v\right): \lambda \in \mathbb{R}\right\}$. Then $\Pi_{M_{n}}$ is an interval projection of $K$, hence $|I P(K)|=\infty$.

For an $l$-dimensional subspace $M \subseteq \mathbb{R}^{d}$ we denote its orthogonal direct complement by $M^{\perp}$. For a vector $v \in \mathbb{R}^{d}$ let $v^{\perp}$ be the hyperplane through the origin that is orthogonal to $v$. We denote the set of all lines in $\mathbb{R}^{d}$ through the origin by $G_{d, 1}$.

Sketch of the proof. Similarly to the proof of Lemma 3.5 one can show that $I P(K)$ is compact. Thus it is enough to show that every element of $I P(K)$ is isolated. We show it by induction on $d$. The statement holds for $d=2$ by Theorem 1.3. Let $d>2$ and $\Pi_{M} \in I P(K)$ for some line $M$. Just as in the proof of Theorem 1.3. we can show that for an endpoint $a$ of the interval $\Pi_{M}(K)$ there exists a unique $w \in K$ such that $\Pi_{M}(w)=a$. Moreover, one can show that there exists a closed convex cone $C \subseteq \mathbb{R}^{d}$ such that $w \in C$ is the apex, $K \subseteq C$ and $a \notin \Pi_{M}(C \backslash w)$. With a similar argument to that in the proof of Theorem[1.3 about fitting pieces, one can show that there exist $\mathbf{i}, \mathbf{j} \in \bigcup_{k=1}^{\infty} \mathcal{I}^{k}$ such that $K_{\mathbf{i}} \cap K_{\mathbf{j}}=\emptyset$ and $\left|\Pi_{M}\left(K_{\mathbf{i}}\right) \cap \Pi_{M}\left(K_{\mathbf{j}}\right)\right|=1$. Let $u \in K_{\mathbf{i}}$ and $v \in K_{\mathbf{j}}$ such that $\Pi_{M}(u)=\Pi_{M}(v)$. Let $N$ be the 2-dimensional linear subspace that contains $M$ and the vector $u-v$ and identify $N$ with $\mathbb{R}^{2}$ such that $M$ is the $x$-axis and $u-v$ is parallel to the $y$-axis. Using similar ideas to those that were used to prove Lemma 3.4 one can show that there exists a neighbourhood $U$ of $M$ in $G_{d, 1}$ such that if $M_{2} \in U$ and $N \cap M_{2}^{\perp}$ is not parallel to $u-v$ then $\Pi_{M_{2}}$ is not an interval projection of $K$ (we note that in this argument we use the fact that $K \subseteq C$ and $\left.a \notin \Pi_{M}(C \backslash w)\right)$. To finish the proof we need to show that there are only finitely many $M_{2} \in I P(K)$ such that $N \cap M_{2}^{\perp}$ is parallel to $u-v$. Notice that if $N \cap M_{2}^{\perp}$ is parallel to $u-v$ then $M_{2} \subseteq(u-v)^{\perp}$ and so $\Pi_{M_{2}}=\Pi_{M_{2}} \circ \Pi_{(u-v)^{\perp}}$. Hence if $\Pi_{M_{2}} \in I P(K)$ then $\Pi_{M_{2}} \in I P\left(\Pi_{(u-v)^{\perp}}(K)\right)$ in $(u-v)^{\perp}=\mathbb{R}^{d-1}$. However, by the inductive assumption $I P\left(\Pi_{(u-v)^{\perp}}(K)\right)$ is a finite set because $\Pi_{(u-v)^{\perp}}(K)$ is a self-similar set since each $S_{i}$ is a homothety.

\section{$5 \quad$ Examples}

For an SS-IFS $\left\{S_{i}\right\}_{i=1}^{m}$ with attractor $K$ we call the unique solution $s$ of the equation

$$
\sum_{i=1}^{m} r_{i}^{s}=1
$$

the similarity dimension of the SS-IFS. A straightforward covering argument shows that $\operatorname{dim}_{H} K \leq s$ and $\mathcal{H}^{s}(K)<\infty$, see for example [6, 5.1 Prop(4)]. Let $0<r<1$ be such that $r_{i} \leq r$ for every $i \in \mathcal{I}$. Then $1=\sum_{i=1}^{m} r_{i}^{s} \leq m \cdot r^{s}$ and hence

$$
\operatorname{dim}_{H} K \leq s \leq \log (m) / \log (1 / r) .
$$

In this section the dimension estimation of the self-similar sets will all be based on this formula, hence we will use it without any reference.

If there exists a compact, convex set $F$ of nonempty interior such that $S_{i}(F) \subseteq F$ for every $i \in \mathcal{I}$ and we define $F_{k}:=\bigcup_{\mathbf{i} \in \mathcal{I}^{k}} S_{\mathbf{i}}(F)$ then $F \supseteq F_{1} \supseteq F_{2} \supseteq \ldots$ and since $K$ is the unique compact attractor of the SS-IFS it follows that $K=\bigcap_{k=1}^{\infty} F_{k}$. Assume furthermore, that $S_{i}$ is a homothety for every $i \in \mathcal{I}$ and for some $\theta \in \mathbb{R}$ every line $L$ that is parallel to $L_{\frac{\pi}{2}+\theta}$ and $L \cap F \neq \emptyset$ we have that $L \cap F_{1} \neq \emptyset$. Then $L \cap F_{k} \neq \emptyset$ for every $k \in \mathbb{N}$ and so $L \cap K \neq \emptyset$. Hence $P_{\theta}(K)=P_{\theta}(F)$ is an interval. It also implies that $\operatorname{dim}_{H} K \geq \operatorname{dim}_{H} P_{\theta}(K)=1$ and $\mathcal{H}^{1}(K) \geq \mathcal{H}^{1}\left(P_{\theta}(K)\right)>0$ because orthogonal projection does not increase the Hausdorff dimension and measure. If for some $\theta \in \mathbb{R}$ we can find such $F$ then we call $F$ a $\theta$-witness of interval projection for the SS-IFS or shortly just say $F$ is a witness for $M=L_{\theta}$. 
Example 5.1. There exists a self-similar 1-set with three interval projections such that the projection intervals have the same length. The 1-dimensional Sierpinski triangle is the attractor of the SS-IFS that contains three homotheties which map an equilateral triangle into itself fixing the corners with similarity ratio $1 / 3$. If $M$ is a line that contains a side $[a, b]$ of the equilateral triangle then $\Pi_{M}(K)=[a, b]$ because the equilateral triangle is a witness for $M$. Hence $I P(K)$ contains at least three projections. It is easy to show that $I P(K)$ contains exactly three projections. See Figure 5.

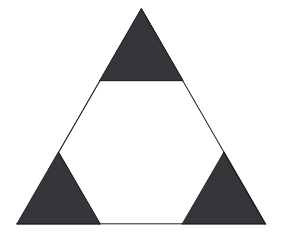

Figure 5 .

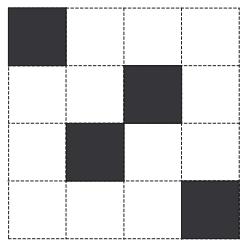

$A+A$
$A+A$

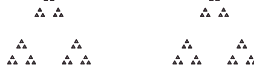

Example 5.2. There exists a self-similar 1-set that has four interval projections. We take four homotheties of similarity ratio $1 / 4$ that map the unit square into itself as it is shown on Figure 6 . The projection of the attractor on both the $x$ - and $y$-axes are intervals of length 1 because the unit square is witness for those. Let $F$ be the rhombus that is the convex hull of the fixed points of the homotheties. Then the homotheties map $F$ into itself fixing the corners. Again $F$ is a witness for the coordinate axes. However, $F$ is a witness for two further lines, see Figure 7 and Figure 8. Checking on $F_{1}$ it is not hard to show that $I P(K)$ consist of exactly four lines.

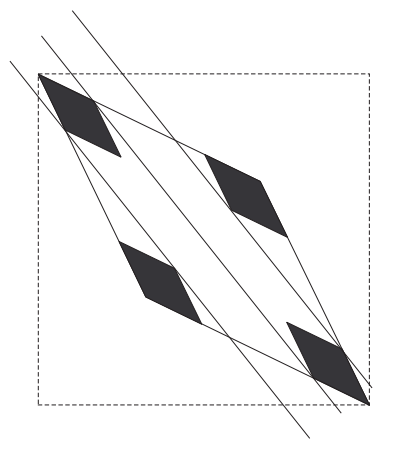

Figure 7.

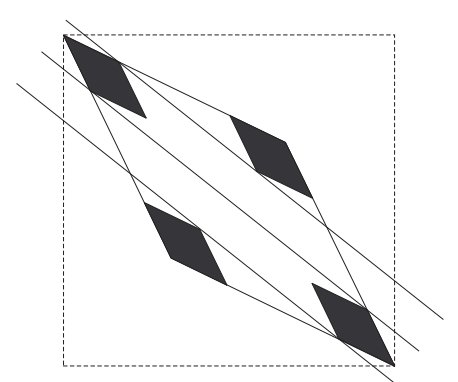

Figure 8.

Remark 5.3. In example 5.2 not all the four projection intervals have the same length. One can easily verify it from that $F$ is a witness for the projections. However, it is also easy to check that $\int x \cdot y \mathrm{~d} \mu(x, y)<0$ when $p$ is the centre of mass of $\left.\mathcal{H}^{1}\right|_{K}$ and $\mu=\left.\mathcal{H}^{1}\right|_{K-p}$. Hence the intervals could not be of same length by Theorem 1.10 .

Example 5.4. There exists a self-similar 1-set with four interval projections such that the projection intervals have the same length. The 1-dimensional four corner set is the attractor of the SS-IFS that contains four homotheties which map the unit square into itself fixing the corners with similarity ratio $1 / 4$. We indicate on Figure 9 what the interval projections are. One can think of Example 5.2 as an affine image of the 1-dimensional four corner set.
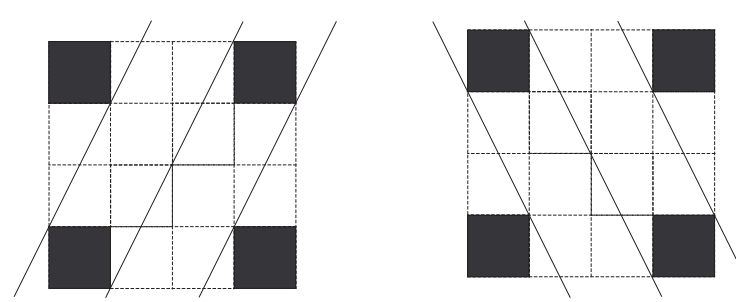

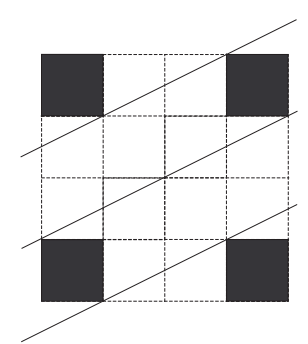

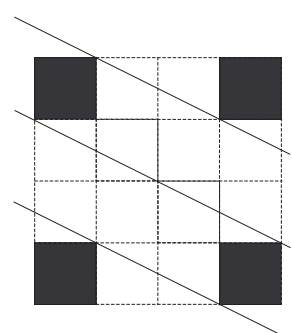

Figure 9.

In the definition of a witness for a line $M=L_{\theta}$ we required the similarities to be homtheties. If the similarities are not all homotheties then we need to understand the intersection of $F$ and $F_{1}$ not only with 
lines that are parallel to $L_{\pi / 2+\theta}$ but also for lines that are parallel to $T\left(L_{\pi / 2+\theta}\right)$ for every $T \in \mathcal{T}$. If we want to show that every projection is an interval then we can define the witness the following way. Let $F$ be a convex compact set with non-empty interior such that $S_{i}(F) \subseteq F$ for every $i \in \mathcal{I}$. Then $F \supseteq F_{1} \supseteq F_{2} \supseteq \ldots$ and the attractor $K=\cap_{k=1}^{\infty} F_{k}$. Assume that every line $L$ that intersects $F$ also intersects $F_{1}$. It follows that every line $L$ that intersects $F$ also intersects $F_{k}$ for every $k \in \mathbb{N}$ and so $L$ intersects $K$. Hence every projection is an interval. If we can find such a set $F$ then we say that $F$ is a witness for every line. Note that for this definition we did not assume that the similarities are homotheties.

Example 5.5. For every $\varepsilon>0$ there exists a totally disconnected self-similar set $K$ of Hausdorff dimension less then $1+\varepsilon$ such that every projection is an interval projection of $K$. Rather than giving a complicated explicit description of the SS-IFS we suggest the maps by drawing pictures. First consider the SS-IFS when the similarities map the unit square into itself by five maps as shown on Figure 10. It satisfies the SSC and the unit square is a witness for every line. Hence every projection is an interval.

To decrease the Hausdorff dimension dimension we take more maps of smaller similarity ratio $r$. We consider a sequence of SS-IFS, again satisfying the SSC, when the similarities map the unit square into itself such that images are close to the diagonals as shown on Figure 11. The number of maps that the SS-IFS consist of is $O(1 / r)$ as $r$ goes to 0 . Thus the similarity dimension approaches 1 as $r$ goes to 0 . Again the unit square is a witness for every line.

In the previous approach the similarities are not homotheties. We can also give examples where every similarity is a homothety. Consider the sequence of SS-IFS, now only satisfying the 'open set condition', when the similarities map the unit square into itself such that the images touch the sides of the unit square as shown on Figure 12. Again the number of maps that the SS-IFS consist of is $O(1 / r)$ and the unit square is a witness for every line.

We note that one could construct an example when both the SSC is satisfied and every similarity is a homothety. However, the constructions is a little bit more complicated to present.

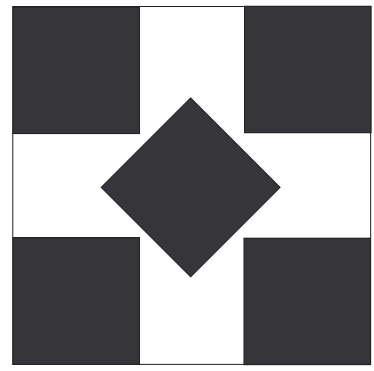

Figure 10.

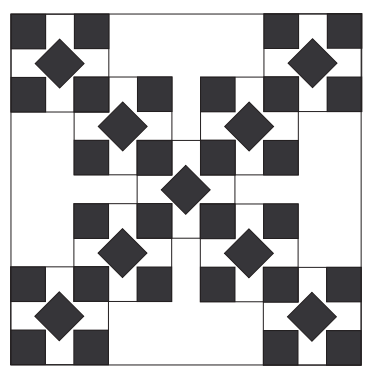

Figure 11.

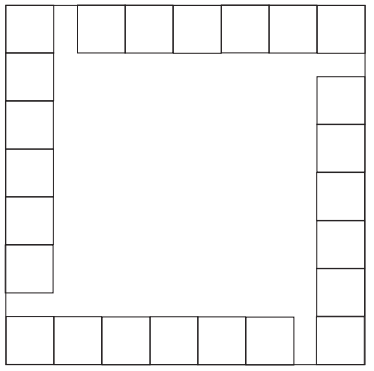

Figure 12.

Example 5.6. There exists a totally disconnected, compact set of Hausdorff dimension 1 such that every projections is an interval projection. For every $n \in \mathbb{N}$ let $\mathcal{I}_{n}$ be an SS-IFS mentioned in Example 5.5 with the Hausdorff dimension of the attractor being less than $1+1 / n$. Let $F$ be the unite square and

$$
F_{n}:=\bigcup\left\{S_{i_{1}} \circ \ldots \circ S_{i_{n}}(F): i_{k} \in \mathcal{I}_{k}, k=1, \ldots, n\right\} .
$$

Then $F_{1} \supseteq F_{2} \supseteq \ldots$ and let $K=\bigcap_{n=1}^{\infty} F_{n}$. By a standard covering argument it is easy to show that $\mathcal{H}^{1+\varepsilon}(K)<\infty$ for every $\varepsilon>0$ and hence $\operatorname{dim}_{H} K \leq 1$. Via an argument similar to the witness of projections one can show that every projection is an interval projection.

Example 5.7. There exists a totally disconnected self-similar set $K$ in $\mathbb{R}^{3}$ such that the projection of $K$ onto every 2-dimensional plane is path connected. Note that if $M \subseteq \mathbb{R}^{3}$ is a 2-dimensional plane, $L \subseteq M$ is a line and $\Pi_{M}(K)$ is connected then $\Pi_{L}(K)$ is an interval because $\Pi_{L} \circ \Pi_{M}=\Pi_{L}$.

In $\mathbb{R}^{3}=\{(x, y, z): x, y, z \in \mathbb{R}\}$ let $C=\left\{(x, y, 0): x^{2}+y^{2}=1\right\}$ be the unit circle and for the sake of this example we say that the points $(1,0,0)$ and $(-1,0,0)$ are the end points of $C$. Let $q>0$ be 'very small' (it will be clear from the construction how small) and let $F=\left\{p \in \mathbb{R}^{3}: \operatorname{dist}(C, p) \leq q\right\}$ be the solid ring. Consider the SS-IFS containing similarities that map $F$ into itself such that the image of the end points of $C$ are mapped into $C$ for every similarity and the union of the images, that is $F_{1}$, form a chain inside $F$, like a necklace, where the images of $F$ are the links. If $q$ is small enough we can do this such that the images, i.e. the links, are disjoint. Hence the strong separation condition is satisfied and so the attractor $K$ 
is totally disconnected. Fix a plane $M$. By taking a sufficient uniformly convergent sequence $f_{n}$ of curves in $\Pi_{M}\left(F_{n}\right)$ one can show that $\Pi_{M}(K)$ is path connected. As in Example 5.5 we can take the similarity ratio $r$ to be very small but still have at most $O(1 / r)$ many similarity in the SS-IFS. Hence we can construct $K$ to have Hausdorff dimension arbitrarily close to 1.

\section{Acknowledgements}

The author was partially supported by an ERC grant (grant number 306494). The author would like to thank Kenneth Falconer for pointing out the connection between the integral of projections and the moment of inertia. The results of the present paper emerged from the author's master thesis research. The author would like to thank Márton Elekes for the help in writing the master thesis.

\section{References}

[1] A. S. Besicovitch, On the fundamental geometrical properties of linearly measurable plane sets of points (III), Math. Ann. 116 (1939), 349-357. MR1513231

[2] K. Falconer, Techniques in Fractal Geometry, John Wiley \& Sons, Ltd., Chichester, 1997. MR1449135 (99f:28013)

[3] K. Falconer and J. M. Fraser, The visible part of plane self-similar sets. Proc. Amer. Math. Soc. 141 (2013), 269-278. MR2988729

[4] Á. Farkas, Projections of self-similar sets with no separation condition. Israel J. Math. 214 (2016), 67-107. MR3540606

[5] H. Federer, The $(\varphi, k)$ rectifiable subsets of n-space, Trans. Amer. Soc. 62, (1947), 114-192. MR0022594 $(9,231 \mathrm{c})$

[6] J. E. Hutchinson, Fractals and self-similarity, Indiana Univ. Math. J. 30 (1981), 713-747. MR0625600 (82h:49026)

[7] R. Kenyon, Projecting the one-dimensional Sierpinski gasket. Israel J. Math. 97 (1997), 221-238. MR1441250 (98i:28002)

[8] J. M. Marstrand, Some fundamental geometrical properties of plane sets of fractional dimensions, Proc. London Math. Soc. (3) 4 (1954), 257-302. MR0063439 (16,121g)

[9] P. Mattila, Geometry of Sets and Measures in Euclidean Spaces, Cambridge University Press, Cambridge, 1995. MR1333890 (96h:28006)

[10] P. Mattila, Hausdorff dimension, orthogonal projections and intersections with planes, Ann. Acad. Sci. Fenn. Ser. A I Math. 1 (1975), 227-244. MR0409774 (53 \#13526)

[11] P. Mattila, Pertti On the structure of self-similar fractals. Ann. Acad. Sci. Fenn. Ser. A I Math. 7 (1982), 189-195. MR0686639 (84j:28011)

[12] A. Schief, Separation properties for self-similar sets, Proc. Amer. Math. Soc. 122 (1994), 111-115. MR1191872 (94k:28012)

[13] JingLing Wang, The open set conditions for graph directed self-similar sets, Random Comput. Dynam. 5 (1997), 283-305. MR1483871 (99g:28019) 